\title{
The prevalence of colistin-resistant Gram-negative bacteria isolated from hospitalized patients with bacteremia
}

\author{
Wichai Santimaleeworagun ${ }^{1,2^{*}}$, Sudaluck Thunyaharn ${ }^{3}$, Piraporn Juntanawiwat ${ }^{4}$, Nachadaporn Thongnoy ${ }^{1}$, \\ Suddhi Harindhanavudhi ${ }^{1}$, Suwapach Nakeesathit ${ }^{1}$, Saowaluk Teschumroon ${ }^{1}$ \\ ${ }^{1}$ Department of Pharmacy, Faculty of Pharmacy, Silpakorn University, Nakhon Pathom 73000, Thailand. \\ ${ }^{2}$ Antibiotic Optimization and Patient Care Project by Pharmaceutical Initiative for Resistant Bacteria and Infectious Diseases Working Group PIRBIG, \\ Silpakorn University, Nakhon Pathom 73000, Thailand. \\ ${ }^{3}$ Faculty of Medical Technology, Nakhonratchasima College, Nakhon Ratchasima 30000, Thailand. \\ ${ }^{4}$ Division of Microbiology, Department of Clinical Pathology, Phramongkutklao Hospital, Bangkok 10400, Thailand.
}

\begin{tabular}{l}
\hline ARTICLE INFO \\
\hline Received on: 05/05/2019 \\
Accepted on: $26 / 10 / 2019$ \\
Available online: $05 / 02 / 2020$
\end{tabular}

\section{Key words:}

Antimicrobial susceptibility, colistin, minimum inhibitory concentration, polymyxins, Gram-negative bacteria.

\begin{abstract}
This study sought to investigate the prevalence of colistin-resistant Gram-negative bacteria (CoR-GNB) among Pseudomonas aeruginosa, Acinetobacter baumannii, Escherichia coli, and Klebsiella pneumoniae isolated from patients with bacteremia and to identify other antimicrobials as a potential therapy for CoR-GNB infections. We retrospectively reviewed the data of non-repeated clinical bacterial isolates from patients admitted to Phramongkutklao Hospital during May 2017-April 2018. We obtained the minimum inhibitory concentrations (MICs) of the studied isolates and interpreted the MIC values followed by the Clinical and Laboratory Standards Institute (CLSI) criteria. Out of 623 bacterial isolates, the prevalence of E. coli was predominantly high (349), followed by K. pneumoniae (150), $P$. aeruginosa (64), and A. baumannii (60). The CoR-GNB rates among E. coli, K. pneumoniae, A. baumannii, and $P$. aeruginosa were $2.9 \%, 17.3 \%, 5.0 \%$, and $1.6 \%$, respectively. Seven out of 26 colistin-resistant $K$. pneumoniae isolates and seven out of 10 colistin-resistant $E$. coli isolates were still susceptible to carbapenems (the MICs for imipenem and meropenem were $\leq 1 \mu \mathrm{g} / \mathrm{ml}$ ). Tigecycline and aminoglycosides might be the best therapeutic choices against CoR-GNB. In conclusion, our findings confirmed a CoR-GNB prevalence of approximately $1.6 \%-17.3 \%$, depending on the bacterial species. Certain available antimicrobials remain effective against CoR-GNB.
\end{abstract}

\section{INTRODUCTION}

Colistin, also called polymixin E, plays an important role as the last line of anti-Gram-negative defense against multidrug resistant (MDR) pathogens, particularly carbapenemresistant Pseudomonas aeruginosa, Acinetobacter baumannii, and Enterobacteriaceae. Colistin is not a new agent; it has been used since the early 1950s. Colistin prescriptions declined in the 1970 s

\section{*Corresponding Author}

Wichai Santimaleeworagun, Department of Pharmacy, Faculty of Pharmacy, Silpakorn University, Nakhon Pathom 73000, Thailand; Antibiotic Optimization and Patient Care Project by Pharmaceutical Initiative for Resistant Bacteria and Infectious Diseases Working Group PIRBIG, Silpakorn University, Nakhon Pathom, 73000 Thailand. E-mail: swichai1234@gmail.com mainly due to its nephrotoxicity. However, the increase of MDR Gram-negative bacteria has led to the revitalized use of colistin during the last decade (Gregoire et al., 2017).

Naturally, some organisms have intrinsic colistin resistance, including Serratia marcescens, Proteus spp., Providencia spp., Morganella morganii, Burkholderia cepacia, and Vibrio cholera (Sherry and Howden, 2018). Unfortunately, because of increasing colistin consumption, organisms that have acquired colistin resistance via chromosomal genes or plasmids have been globally reported. Overall, colistin resistance among non-inherent resistant bacteria in Enterobacteriaceae has a prevalence of approximately $0.67 \%-1.6 \%$, with high rates in Enterobacter spp. (13.9\%-20.1\%), followed by K. pneumoniae $(1.5 \%-6.8 \%)$ and E. coli $(0.2 \%-0.6 \%)$ (Sherry and Howden, 2018).

For non-fermentative Gram-negative bacteria, the rates of colistin-resistant A. baumannii in the global SENTRY 
Antimicrobial Surveillance database from 2001 to 2011 ranged from $0.9 \%$ to $3.3 \%$ (Gales et al., 2011). Recently, in data from the China Surveillance of Antimicrobial Resistance Program during 2009-2014, the rate of non-susceptible $A$. baumannii increased slightly from $0.9 \%$ to $3.0 \%$ over a 6 -year period (Gao et al., 2017). Conversely, the rates of colistin resistance in $P$. aeruginosa in SENTRY from US and European hospitals during 2009-2012 remained at approximately $0.3 \%$ (Sader et al., 2014). However, the results of the Canadian Ward surveillance study reported colistin resistance in up to $5.1 \%$ of P. aeruginosa isolates obtained from 2008 to 2015 (Walkty et al., 2017).

In one study from the largest national tertiary referral center in Thailand, Eiamphungporn et al. (2018) found that among 317 study isolates, colistin resistance [minimum inhibitory concentration (MIC) $>2 \mathrm{mg} / \mathrm{l}]$ was observed in 226 isolates (71.3\%), including 13 E. coli and $213 \mathrm{~K}$. pneumoniae isolates.

The impact of colistin-resistant Gram-negative bacteria (CoR-GNB) on clinical outcomes is evident. Infection due to colistin-resistant $K$. pneumoniae was significantly associated with an increased risk for in-hospital mortality (Rojas et al., 2017). Thus, an appropriate regimen against CoR-GNB is urgently required.

To date, data on salvage therapy for CoR-GNB infections are limited. In addition, CoR-GNB has been reported sporadically at our institute, and there is an absence of new agents effective against such organisms. Therefore, this study sought to study the prevalence of CoR-GNB among $P$. aeruginosa, A. baumannii, E. coli, and K. pneumoniae isolated from patients with bacteremia and to determine the remaining antibacterial agents displaying activity against CoR-GNB isolates.

\section{MATERIALS AND METHODS}

\section{Study design}

This study retrospectively reviewed the data of nonrepeated clinical bacterial isolates from patients admitted to Phramongkutklao Hospital, a 1,200-bed tertiary care center in Bangkok, Thailand during May 2017-April 2018. This study was approved by the ethical review committee of the Royal Thai Army Medical Department (approval no. Q005h/61 Exp) and was permitted by the Director of the Phramongkutklao Hospital prior to data collection.

\section{Bacterial isolates}

All clinical isolates obtained from patients with bacteremia were included in this study. We obtained the results for the first isolate of P. aeruginosa, A. baumannii, E. coli, or K. pneumoniae from each patient. We excluded all isolates if the follow-up isolates with different resistance patterns less than two antibiotic agents during 14 days after isolation of the first strain.

\section{Definitions}

MDR Gram-negative bacteria were those bacteria non-susceptible to at least three antimicrobial groups among aminoglycosides, antipseudomonal penicillins, third- or fourth-generation cephalosporins, carbapenems, trimethoprimsulfamethoxazole, and fluoroquinolones. Third-generation cephalosporin non-susceptible Gram-negative bacteria included the studied isolates of $E$. coli and $K$. pneumoniae that were non-susceptible to cefotaxime, ceftriaxone, or ceftazidime.
Carbapenem-resistant (CR) Gram-negative bacteria included the studied isolates of $E$. coli and $K$. pneumoniae that were non-susceptible to any carbapenems or the $P$. aeruginosa and $A$. baumannii isolates that were non-susceptible to imipenem, meropenem, or doripenem. CoR-GNB included the studied isolates with an MIC $\geq 4 \mathrm{~g} / \mathrm{ml}$ to colistin in the broth microdilution method according to the Clinical and Laboratory Standards Institute guidelines, version 2019, which provide antimicrobial susceptibility information for methods, condition testing, validation, and interpretation (CLSI, 2019).

\section{Data collection}

We determined the MIC of the non-repeated clinical isolates. The MIC of the antimicrobial agents was determined using automated susceptibility testing (Thermo Scientific ${ }^{\mathrm{TM}}$ Sensititre ${ }^{\mathrm{TM}}$ ARISTM $2 X$ Instrument) based on the broth microdilution method (Chew et al., 2017). Growth was determined by fluorescence measurement after 18-24 hours of incubation depending on the species. The MIC value of the antimicrobials in each strain was interpreted as susceptible, intermediate, or resistant using the CLSI breakpoint.

Descriptive statistics were used for the MIC distribution. Our study investigated the MIC values of antimicrobial agents against the studied isolates. The MIC50 and MIC90 values were defined as the lowest concentration of antimicrobials at which $50 \%$ and $90 \%$ of the isolates were inhibited, respectively.

\section{RESULTS}

During the 1-year study period, 623 studied bacterial isolates were obtained from blood cultures including $349 \mathrm{E}$. coli isolates, $150 \mathrm{~K}$. pneumoniae isolates, $60 \mathrm{~A}$. baumannii isolates, and $64 P$. aeruginosa isolates. Forty out of 623 isolates $(6.4 \%)$ were CoR-GNB. The CoR-GNB prevalence rates among E. coli, $K$. pneumoniae A. baumannii, and $P$. aeruginosa were $2.9 \%$, $17.3 \%, 5.0 \%$, and $1.6 \%$, respectively (Fig. 1).

Seven out of the 26 clinical colistin-resistant $K$. pneumoniae isolates were still susceptible to carbapenems (imipenem $\leq 2 \mu \mathrm{g} / \mathrm{ml}$ and meropenem $\leq 2 \mu \mathrm{g} / \mathrm{ml}$ ). Conversely, the remaining 19 colistin-resistant $K$. pneumoniae isolates were susceptible to amikacin, gentamicin, and tigecycline with MIC 50/90 (MIC range) values of $16 / 16(\leq 8-32) \mu \mathrm{g} / \mathrm{ml}, 2 / 4(\leq 2->8) \mu \mathrm{g} / \mathrm{ml}$,

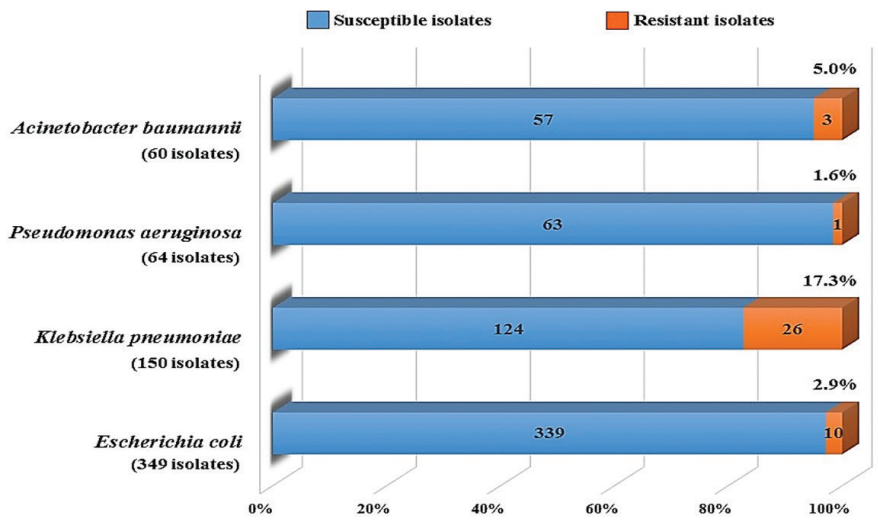

Figure 1. Colistin resistance was identified in $2.9 \%, 17.3 \%, 1.6 \%$, and $5.0 \%$ of E. coli $(n=349), K$. pneumoniae $(n=150)$, P. aeruginosa $(n=64)$, and A. baumannii $(n=60)$ isolates, respectively. 
Table 1. MIC distribution and susceptible rate based on species-susceptible breakpoint among colistin-resistant Gram-negative bacteria ( $N=40$ isolates).

\begin{tabular}{|c|c|c|c|c|c|c|c|c|}
\hline \multirow{2}{*}{ Tigecycline } & \multicolumn{7}{|c|}{$\operatorname{MIC}(\mu \mathrm{g} / \mathrm{ml})$} & \multirow{2}{*}{$\begin{array}{l}\text { Susceptible rate (\%) } \\
\leq 2 \mu \mathrm{g} / \mathrm{ml} / \leq 1 \mu \mathrm{g} / \mathrm{ml}\end{array}$} \\
\hline & $\leq 0.25$ & 0.5 & & 1 & 2 & 4 & 8 & \\
\hline K. pneumoniae $(n=26)$ & 2 & 17 & & 5 & 1 & 1 & - & $96.2 / 92.3$ \\
\hline E. $\operatorname{coli}(n=10)$ & 4 & 3 & & 2 & 1 & - & - & $100 / 90$ \\
\hline A. baumannii $(n=3)$ & 2 & - & & - & - & 1 & - & $66 / 66$ \\
\hline P. aeruginosa $(n=1)$ & \multicolumn{7}{|c|}{ N/A } & N/A \\
\hline \multirow{2}{*}{ Imipenem } & \multicolumn{7}{|c|}{ MIC $(\mu \mathrm{g} / \mathrm{ml})$} & \multirow{2}{*}{ Susceptible rate $(\%)$} \\
\hline & $\leq 0.5$ & 1 & & 2 & 4 & 8 & $>8$ & \\
\hline K. pneumoniae $(n=26)$ & 5 & 2 & & - & - & - & 19 & $26.9 ;(\leq 1 \mathrm{ug} / \mathrm{ml})$ \\
\hline E. $\operatorname{coli}(n=10)$ & 7 & - & & - & - & - & 3 & $70 ;(\leq 1 \mathrm{ug} / \mathrm{ml})$ \\
\hline A. baumannii $(n=3)$ & 1 & - & & - & - & - & 2 & $33.3 ;(\leq 2 \mu \mathrm{g} / \mathrm{ml})$ \\
\hline P. aeruginosa $(n=1)$ & - & 1 & & - & - & - & - & $100 ;(\leq 2 \mu \mathrm{g} / \mathrm{ml})$ \\
\hline \multirow{2}{*}{ Meropenem } & \multicolumn{7}{|c|}{ MIC $(\mu \mathrm{g} / \mathrm{ml})$} & \multirow{2}{*}{ Susceptible rate $(\%)$} \\
\hline & $\leq 0.5$ & 1 & & 2 & 4 & 8 & $>8$ & \\
\hline K. pneumoniae $(n=26)$ & 7 & - & & - & - & - & 19 & $26.9 ;(\leq 1 \mathrm{ug} / \mathrm{ml})$ \\
\hline E. $\operatorname{coli}(n=10)$ & 6 & 1 & & - & - & - & 3 & $70 ;(\leq 1 \mathrm{ug} / \mathrm{ml})$ \\
\hline A. baumannii $(n=3)$ & 1 & - & & - & - & - & 2 & $33.3 ;(\leq 2 \mu \mathrm{g} / \mathrm{ml})$ \\
\hline P. aeruginosa $(n=1)$ & 1 & - & & - & - & - & - & $100 ;(\leq 2 \mu \mathrm{g} / \mathrm{ml})$ \\
\hline \multirow{2}{*}{ Ciprofloxacin } & \multicolumn{7}{|c|}{ MIC $(\mu \mathrm{g} / \mathrm{ml})$} & \multirow{2}{*}{ Susceptible rate $(\%)$} \\
\hline & $\leq 0.06$ & 0.25 & & 0.5 & 1 & 2 & $>2$ & \\
\hline K. pneumoniae $(n=26)$ & - & - & & 2 & - & - & 24 & $0 ;(\leq 0.25 \mathrm{ug} / \mathrm{ml})$ \\
\hline E. $\operatorname{coli}(n=10)$ & 1 & 3 & & - & 1 & 1 & 4 & $40 ;(\leq 0.25 \mathrm{ug} / \mathrm{ml})$ \\
\hline A. baumannii $(n=3)$ & 1 & - & & - & - & 1 & 1 & $33.3 ;(\leq 1 \mathrm{ug} / \mathrm{ml})$ \\
\hline P. aeruginosa $(n=1)$ & - & - & & - & - & - & 1 & $0 ;(\leq 0.5 \mathrm{ug} / \mathrm{ml})$ \\
\hline \multirow{2}{*}{ Amikacin } & \multicolumn{7}{|c|}{$\mathrm{MIC}(\mu \mathrm{g} / \mathrm{ml})$} & \multirow{2}{*}{$\begin{array}{c}\text { Susceptible rate (\%) } \\
\leq 16 \mu \mathrm{g} / \mathrm{ml}\end{array}$} \\
\hline & $\leq 8$ & & 16 & & 32 & & $>32$ & \\
\hline K. pneumoniae $(n=26)$ & 9 & & 13 & & 3 & & 1 & 84.6 \\
\hline E. $\operatorname{coli}(n=10)$ & 7 & & 1 & & 2 & & - & 80 \\
\hline A. baumannii $(n=3)$ & 1 & & - & & 1 & & 1 & 33.3 \\
\hline P. aeruginosa $(n=1)$ & 1 & & - & & - & & - & 100 \\
\hline \multirow{2}{*}{ Gentamicin } & \multicolumn{7}{|c|}{$\operatorname{MIC}(\mu \mathrm{g} / \mathrm{ml})$} & Susceptible rate $(\%)$ \\
\hline & $\leq 2$ & & 4 & & 8 & & $>8$ & $\leq 4 \mu \mathrm{g} / \mathrm{ml}$ \\
\hline$K$. pneumoniae $(n=26)$ & 21 & & 1 & & 1 & & 3 & 84.6 \\
\hline E. $\operatorname{coli}(n=10)$ & 5 & & - & & - & & 5 & 50 \\
\hline A. baumannii $(n=3)$ & 1 & & - & & - & & 2 & 33.3 \\
\hline P. aeruginosa $(n=1)$ & - & & - & & - & & 1 & 0 \\
\hline
\end{tabular}

and $0.5 / 1.0(0.5-2) \mu \mathrm{g} / \mathrm{ml}$, respectively. Of the 10 colistin-resistant $E$. coli isolates, seven were susceptible to carbapenems (imipenem $\leq 1 \mu \mathrm{g} / \mathrm{ml}$ and meropenem $\leq 1 \mu \mathrm{g} / \mathrm{ml}$ ), whereas the remaining three carbapenem non-susceptible isolates were universally susceptible to tigecycline with an MIC range of $0.5-1 \mu \mathrm{g} / \mathrm{ml}$ (Table 1).

One of the three clinical colistin-resistant $A$. baumannii isolates and one of the colistin-resistant $P$. aeruginosa isolates were carbapenem (imipenem and meropenem) susceptible. One of the two colistin-resistant $A$. baumannii isolates was susceptible to tigecycline. The MIC distribution of the antimicrobials is shown in Table 1.

\section{DISCUSSION}

Polymyxins are used as last-resort antibiotics in many areas with MDR Gram-negative occurrence; however, this has currently resulted in polymyxin-resistant bacteria. For this reason, the prevalence of colistin resistance among Gram-negative clinical isolates in Thailand was evaluated; however, only E. coli, $K$. pneumoniae, $P$. aeruginosa, and $A$. baumannii were included. At our setting, a prevalence of $6.4 \%$ was reported for non-repeated CoR-GNB isolates obtained from blood cultures. Among them, $K$. pneumoniae was the most common genus associated with polymyxin resistance $(17.3 \%)$, followed by $E$. coli with a prevalence rate of $2.9 \%$. Overall, the high rate of colistin resistance observed in $K$. pneumoniae was consistent with a previous study that showed higher rates of colistin resistance in $K$. pneumoniae than in E. coli (Sherry and Howden, 2018). However, the prevalence of colistinresistant $K$. pneumoniae in our setting was much higher than rates reported in previous studies. This may have occurred because our studied isolates were largely CR strains. A high proportion of colistin resistance among CR Enterobacteriaceae strains has been reported worldwide (Sader et al., 2015). 
Not surprisingly, the prevalence of colistin resistance among $A$. baumannii and $P$. aeruginosa isolates in this study remained relatively low, similar to the reported rates from other studies (A. baumannii and $2.4 \%-4.8 \%$ and $P$. aeruginosa $0 \%$ $1.4 \%$ ). Additionally, even meropenem-non-susceptible, MDR, and extensively drug resistant (XDR) Pseudomonas aeruginosa and A. baumannii isolates have maintained low colistin resistance rates (Sherry and Howden, 2018).

Until now, CoR-GNB infections have been extremely associated with high mortality (Capone et al., 2013; Lertsrisatit et al., 2017). The therapeutic choices against such pathogens require investigation. The results of this study showed that overall characteristics of colistin-resistant Gram-negative bacteria include susceptibility rates of $92.5 \%, 80.0 \%$, and $70.0 \%$ to tigecycline (MIC susceptible breakpoint $\leq 2 \mu \mathrm{g} / \mathrm{ml}$ ), amikacin (MIC $\leq 16 \mu \mathrm{g} /$ $\mathrm{ml}$ ), and gentamicin ( $\mathrm{MIC} \leq 4 \mu \mathrm{g} / \mathrm{ml}$ ), respectively. These results are consistent with previous reports showing that colistin-resistant strains displayed susceptibility rates of $75.00 \%$ and $29.17 \%$ to tigecycline and amikacin, respectively (Arjun et al., 2017). This finding was consistent with our previous study on colistinresistant $A$. baumannii, which revealed that all strains were sensitive to tigecycline (Lertsrisatit et al., 2017). Thus, tigecycline and aminoglycosides might be options for CoR-GNB. Moreover, Prawang et al. (2019) showed that a tigecycline-gentamicin combination could suppress clinical isolates of colistin-resistant $K$. pneumoniae at low concentrations. In some cases of serious infection due to CoR-GNB infection, this combination might be useful in clinical therapy. However, some beta-lactams are able to cover certain strains of $A$. bamannii or $P$. aeruginosa. They could be an alternative choice for patients with bloodstream infections because tigecycline has a very low concentration in the serum.

\section{CONCLUSION}

Our finding confirmed the presence of CoR-GNB in Thailand. The CoR-GNB prevalence rates among E. coli, $K$. pneumoniae, A. baumannii, and $P$. aeruginosa were $2.9 \%, 17.3 \%$, $5.0 \%$, and $1.6 \%$, respectively. The highest rate of colistin-resistant pathogens was predominately found in K. pneumoniae. Therefore, the appropriate colistin use accompanied by the shortest duration of treatment may minimize the troublesome situation of colistin resistance. As a last resort, tigecycline showed the best in vitro activity against CoR-GNB.

\section{ACKNOWLEDGMENTS}

We would like to thank the Faculty of Pharmacy, Silpakorn University for providing the research budget.

\section{CONFLICT OF INTEREST}

Authors declare that they have no conflicts of interest.

\section{REFERENCES}

Arjun R, Gopalakrishnan R, Nambi PS, Kumar DS, Madhumitha R, Ramasubramanian V. A study of 24 patients with colistin-resistant Gramnegative isolates in a tertiary care hospital in South India. Indian J Crit Care Med, 2017; 21:317-21.

Capone A, Giannella M, Fortini D, Giordano A, Meledandri M, Ballardini M, Venditti M, Bordi E, Capozzi D, Balice MP, Tarasi A, Parisi G, Lappa A, Carattoli A, Petrosillo N. High rate of colistin resistance among patients with carbapenem-resistant Klebsiella pneumoniae infection accounts for an excess of mortality. Clin Microbiol Infect, 2013; 19:E23-30.
Chew KL, La MV, Lin RTP, Teo JWP. Colistin and polymyxin b susceptibility testing for carbapenem-resistant and mor-positive Enterobacteriaceae: comparison of Sensititre, Microscan, Vitek 2, And Etest with broth microdilution. J Clin Microbiol, 2017; 55:2609-16.

Clinical and Laboratory Standard Institute. Performance standards for antimicrobial susceptibility testing. CLSI supplement M100. 29th edition, CLSI, Wayne, PA, 2019.

Eiamphungporn W, Yainoy S, Jumderm C, Tan-Arsuwongkul R, Tiengrim S, Thamlikitkul V. Prevalence of the colistin resistance gene mcr1 in colistin-resistant Escherichia coli and Klebsiella pneumoniae isolated from humans in Thailand. J Glob Antimicrob Resist, 2018; 15:32-5.

Gales AC, Jones RN, Sader HS. Contemporary activity of colistin and polymyxin B against a worldwide collection of Gram-negative pathogens: results from the SENTRY Antimicrobial Surveillance Program (2006-09). J Antimicrob Chemother, 2011; 66:2070-4.

Gao L, Lyu Y, Li Y. Trends in drug resistance of Acinetobacter baumannii over a 10-year period: nationwide data from the China surveillance of antimicrobial resistance program. Chin Med J (Engl), 2017; 130:659-64.

Gregoire N, Aranzana-Climent V, Magreault S, Marchand S, Couet W. Clinical pharmacokinetics and pharmacodynamics of colistin Clin Pharmacokinet, 2017; 56:1441-60.

Lertsrisatit Y, Santimaleeworagun W, Thunyaharn S, Traipattanakul J. In vitro activity of colistin mono- and combination therapy against colistin-resistant Acinetobacter baumannii, mechanism of resistance, and clinical outcomes of patients infected with colistin-resistant A. baumannii at a Thai university hospital. Infect Drug Resist, 2017; 10:437-43.

Prawang A, Santimaleeworagun W, Changpradub D, Thunyaharn S, Puttilerpong C. In vitro antibiotic synergy colistin-resistant Klebsiella pneumoniae. Southeast Asian J Trop Med Public Health, 2019; 50(4):703-7.

Rojas LJ, Salim M, Cober E, Richter SS, Perez F, Salata RA, Kalayjian RC, Watkins RR, Marshall S, Rudin SD, Domitrovic TN, Hujer AM, Hujer KM, Doi Y, Kaye KS, Evans S, Fowler VG Jr, Bonomo RA, van Duin D. Colistin resistance in carbapenem-resistant Klebsiella pneumoniae: laboratory detection and impact on mortality. Clin Infect Dis, 2017; 64:711-8.

Sader HS, Castanheira M, Flamm RK, Mendes RE, Farrell DJ, Jones RN. Tigecycline activity tested against carbapenem-resistant Enterobacteriaceae from 18 European nations: results from the SENTRY surveillance program (2010-2013). Diagn Microbiol Infect Dis, 2015; 83:183-6.

Sader HS, Farrell DJ, Flamm RK, Jones RN. Antimicrobial susceptibility of Gram-negative organisms isolated from patients hospitalised with pneumonia in US and European hospitals: results from the SENTRY Antimicrobial Surveillance Program, 2009-2012. Int J Antimicrob Agents, 2014; 43:328-34.

Sherry N, Howden B. Emerging Gram negative resistance to last-line antimicrobial agents fosfomycin, colistin and ceftazidimeavibactam - epidemiology, laboratory detection and treatment implications. Expert Rev Anti Infect Ther, 2018; 16:289-306.

Walkty A, Lagace-Wiens P, Adam H, Baxter M, Karlowsky J, Mulvey MR, McCracken M, Zhanel GG. Antimicrobial susceptibility of 2906 Pseudomonas aeruginosa clinical isolates obtained from patients in Canadian hospitals over a period of 8 years: results of the Canadian Ward surveillance study (CANWARD), 2008-2015. Diagn Microbiol Infect Dis, $2017 ; 87: 60-3$.

How to cite this article:

Santimaleeworagun W, Thunyaharn S, Juntanawiwat P, Thongnoy N, Harindhanavudhi S, Nakeesathit S, Teschumroon $\mathrm{S}$. The prevalence of colistin-resistant Gram-negative bacteria isolated from hospitalized patients with bacteremia. J Appl Pharm Sci, 2020; 10(02):056-059. 